\title{
Predictors of Clinically Significant Echocardiography Findings in Older Adults with Syncope: A Secondary Analysis
}

\author{
Marc A. Probst, MD, MS*, Thomas A. Gibson, BSc², Robert E. Weiss, PhD², Annick N. Yagapen, MPH, CCRP3, \\ Susan E. Malveau, MSBE${ }^{3}$, David H. Adler, MD, MPH${ }^{4}$, Aveh Bastani, MD ${ }^{5}$, Christopher W. Baugh, MD, MBA 6 , \\ Jeffrey M. Caterino, MD, MPH${ }^{7}$, Carol L. Clark, MD, MBA ${ }^{8}$, Deborah B. Diercks, MD, MPH , Judd E. Hollander, MD ${ }^{10}$, \\ Bret A. Nicks, MD, MHA ${ }^{11}$, Daniel K. Nishijima, MD, MAS ${ }^{12}$, Manish N. Shah, MD, MPH ${ }^{13}$, Kirk A. Stiffler, MD ${ }^{14}$, \\ Alan B. Storrow, MD ${ }^{15}$, Scott T. Wilber, MD ${ }^{14}$, Benjamin C. Sun, MD, MPP 3
}

\begin{abstract}
'Department of Emergency Medicine, Mount Sinai School of Medicine, New York, New York; ${ }^{2}$ Department of Biostatistics, University of California, Los Angeles, California; ${ }^{3}$ Center for Policy and Research in Emergency Medicine, Department of Emergency Medicine, Oregon Heath \& Science University, Portland, Oregon; ${ }^{4}$ Department of Emergency Medicine, University of Rochester, New York; ${ }^{5}$ Department of Emergency Medicine, William Beaumont Hospital-Troy, Troy, Michigan; ${ }^{\circ}$ Department of Emergency Medicine, Brigham \& Women's Hospital, Boston, Massachusetts; ${ }^{7}$ Department of Emergency Medicine, The Ohio State University Wexner Medical Center, Columbus, Ohio; ${ }^{8}$ Department of Emergency Medicine, William Beaumont Hospital-Royal Oak, Royal Oak, Michigan; ' Department of Emergency Medicine, University of Texas-Southwestern, Dallas, Texas; ${ }^{10}$ Department of Emergency Medicine, Thomas Jefferson University Hospital, Philadelphia, Pennsylvania; ${ }^{11}$ Department of Emergency Medicine, Wake Forest School of Medicine, Winston Salem, North Carolina; ${ }^{12}$ Department of Emergency Medicine, UC Davis School of Medicine, Sacramento, California; ${ }^{13}$ Department of Emergency Medicine, University of Wisconsin-Madison, Madison, Wisconsin; ${ }^{14}$ Department of Emergency Medicine, Northeastern Ohio Medical University, Rootstown, Ohio; ${ }^{15}$ Department of Emergency Medicine, Vanderbilt University, Nashville, Tennessee.
\end{abstract}

BACKGROUND: Syncope is a common reason for visiting the emergency department (ED) and is associated with significant healthcare resource utilization.

OBJECTIVE: To develop a risk-stratification tool for clinically significant findings on echocardiography among older adults presenting to the ED with syncope or nearsyncope.

DESIGN: Prospective, observational cohort study from April 2013 to September 2016

SETTING: Eleven EDs in the United States

PATIENTS: We enrolled adults ( $\geq 60$ years) who presented to the ED with syncope or near-syncope who underwent transthoracic echocardiography (TTE).

MEASUREMENTS: The primary outcome was a clinically significant finding on TTE. Clinical, electrocardiogram, and laboratory variables were also collected. Multivariable logistic regression analysis was used to identify predictors of significant findings on echocardiography.

RESULTS: A total of 3,686 patients were enrolled. Of these, 995 (27\%) received echocardiography, and $215(22 \%)$ had a significant finding on echocardiography. Regression analysis identified five predictors of significant findings: (1) history of congestive heart failure, (2) history of coronary artery disease, (3) abnormal electrocardiogram, (4) high-sensitivity troponin- $\mathrm{T}>14 \mathrm{pg} / \mathrm{mL}$, and 5) N-terminal pro B-type natriuretic peptide $>125 \mathrm{pg} / \mathrm{mL}$. These five variables make up the ROMEO (Risk Of Major Echocardiography findings in Older adults with syncope) criteria. The sensitivity of a ROMEO score of zero for excluding significant findings on echocardiography was $99.5 \%$ (95\% Cl: 97.4\%-99.9\%) with a specificity of $15.4 \%$ (95\% Cl: $13.0 \%-18.1 \%)$.

CONCLUSIONS: If validated, this risk-stratification tool could help clinicians determine which syncope patients are at very low risk of having clinically significant findings on echocardiography.

FUNDING: This project was supported by the National Heart, Lung, and Blood Institute of the National Institutes of Health under Award Number R01 HL111033. Dr. Probst is supported by the National Heart, Lung, and Blood Institute of the National Institutes of Health under Award Number K23HL132052-02. . Journal of Hospital Medicine 2018;13:823-828. Published online first September 26, 2018. () 2018 Society of Hospital Medicine

\footnotetext{
*Address for correspondence and reprint requests: Marc A. Probst, MD, MS, Department of Emergency Medicine, Mount Sinai School of Medicine, 3 East 101st Street, Second Floor, Rm 218, New York, NY 10029; Telephone: 212-8248094; Fax: 212- 426-1946; E-mail: mprobst@gmail.com

Additional Supporting Information may be found in the online version of this article.
}

Received: April 19, 2018; Revised: July 12, 2018; Accepted: July 27, 2018

() 2018 Society of Hospital Medicine DOI 10.12788/jhm.3082 yncope, defined as a transient loss of consciousness and postural tone followed by complete, spontaneous return to neurological baseline, accounts for over one million (or approximately $1 \%$ ) of all emergency department (ED) visits per year in the United States (US). ${ }^{12}$ Given the breadth of etiologies for syncope, including certain life-threatening conditions, extensive diagnostic evaluation and hospitalization for this complaint is common. ${ }^{3.7}$ The estimated costs of syncope-related hospitalizations are over $\$ 2.4$ billion annually in the US. ${ }^{8}$ 
The 2011 American College of Cardiology Foundation appropriate use criteria for echocardiography state that syncope is an appropriate indication for transthoracic echocardiography (TTE) even when there are no other symptoms or signs of cardiovascular disease. ${ }^{9}$ This broad recommendation may be appropriate since a finding of severe valvular disease would generally merit consultation with a cardiothoracic surgeon to assess the potential for surgical intervention. ${ }^{10}$ However, routine use of echocardiogram in all syncope patients could result in increased healthcare costs, patient discomfort, and incidental findings of unclear significance, while rarely changing diagnosis or management. ${ }^{11,12}$

In an attempt to reduce potentially unnecessary TTE testing, several studies have tried to identify patients at very low risk of structural heart disease. ${ }^{13-17}$ These investigations suggest that TTE is not indicated in syncope patients with a normal ECG and a normal cardiac exam. However, this literature is limited by retrospective study design and/or small sample sizes. The 2017 American Heart Association/American College of Cardiology/ Heart Rhythm Society syncope guidelines recommend TTE for a patient in whom structural heart disease is suspected, but they are not explicit about how to make this determination. ${ }^{18}$ Thus, it is still unclear which syncope patients require TTE since a standardized approach to assessing risk of clinically significant findings on TTE has not yet been rigorously developed.

The objective of this study was to develop a risk-stratification tool to identify older adults at very low risk of having a major, clinically significant finding on rest TTE after presenting to the ED with syncope or near-syncope. Using clinical, ECG, and cardiac biomarker data, we created the ROMEO (Risk Of Major Echocardiography findings in Older adults with syncope) score to help optimize resource utilization for syncope.

\section{METHODS}

\section{Study Design and Setting}

We conducted a large, multicenter, prospective, observational cohort study of older adults who presented to an ED with syncope or near-syncope (ClinicalTrials.gov identifier: NCT01802398). The study was approved by the institutional review boards at all sites and written informed consent was obtained from all participating subjects. The study was conducted at 11 academic EDs across the US (Appendix Table 1).

\section{Study Population}

Patient inclusion criteria for eligibility were age $\geq 60$ years with a complaint of syncope or near-syncope. Syncope was defined as transient loss of consciousness, associated with postural loss of tone, with immediate, spontaneous, and complete recovery. Near syncope was defined as the sensation of imminent loss of consciousness. Patients were excluded if their symptoms were thought to be due to intoxication, seizure, stroke, head trauma, or hypoglycemia. Additional exclusion criteria were the need for medical intervention to restore consciousness (eg, defibrillation), new or worsening confusion, and inability to obtain informed consent from the patient or a legally authorized representative.

This analysis included only patients who received a TTE during the index visit (either in the ED, observation unit, or while admit- ted to the hospital). This dataset was also used for other analyses addressing questions relevant to the ED management of syncope.

\section{Measurements}

All patients underwent a standardized history, physical examination, laboratory, and 12-lead ECG testing. Trained research assistants (RA) directly queried patients about symptoms associated with the syncopal episode. Data on the patient's past medical history, medications, and physical examination findings were collected prospectively from treating providers.

Research staff obtained blood samples for testing at a core laboratory (University of Rochester, Rochester, NY). Two assays were performed using the Roche Elecsys platform: N-terminal pro B-type natriuretic peptide (NT-proBNP) and the 5th generation high-sensitivity cardiac troponin $\mathrm{T}$ (hs- $\mathrm{TnT}$ ). NT-proBNP was classified as abnormal above a cutoff of $125 \mathrm{pg} / \mathrm{mL}$. HsTnT was classified as abnormal above the 99th percentile for a reference population (14 pg/mL). Although hs-TnT was not approved by the U.S. Food and Drug Administration (FDA) at the time of the study, we anticipated that this assay would receive approval and be integrated into future standard of care (FDA approval was granted in January 2017). Rest TTEs were ordered at the discretion of the treating providers.

\section{Outcome Measures}

The primary outcome for this secondary analysis was a major, clinically significant finding on TTE. ${ }^{13,14,16,19}$ These included severe aortic stenosis $\left(<1 \mathrm{~cm}^{2}\right)$, severe mitral stenosis, severe aortic/mitral regurgitation, reduced ejection fraction (defined either quantitatively as less than $45 \%$ or qualitatively as "severe left ventricular dysfunction"), hypertrophic cardiomyopathy with outflow tract obstruction, severe pulmonary hypertension, right ventricular dysfunction/strain, large pericardial effusion, atrial myxoma, or regional wall motion abnormalities.

All echocardiogram reports were independently reviewed by two research physicians. Discrepant reviews were resolved by the research physicians and two of the study investigators (BS, CB). Of note, all the TTEs obtained were formal echocardiographic studies, not bedside ultrasonography performed by the emergency physician.

\section{Candidate Predictors}

Potential candidate predictors were identified through a prior expert panel process. ${ }^{20,21}$ Candidate predictors included age, sex, abnormal heart sounds, exertional syncope, shortness of breath, chest pain, near-syncope, family history of sudden cardiac death, high (>180 mm Hg) or low (<90 mm Hg) systolic blood pressure, abnormal ECG, elevated hs-TnT, elevated NT-proBNP, and history of the following: hypertension, cardiac dysrhythmia, renal failure, diabetes, congestive heart failure (CHF), and coronary artery disease (CAD).

The first obtained ECG was abstracted by one of five research study physicians blinded to all clinical data. Research study physicians demonstrated high interrater reliability (kappa $>0.80$ ) in distinguishing normal from abnormal ECGs in a training set of 50 ECGs. Abnormal ECG interpretations included 
nonsinus rhythms (including paced rhythms), multiple premature ventricular complexes, sinus bradycardias ( $\leq 40 \mathrm{bpm}$ ), ventricular hypertrophies, short PR segment intervals ( $<100$ milliseconds [ms]), axis deviations, first degree blocks ( $>200 \mathrm{~ms})$, complete bundle branch blocks, Brugada patterns, Wolff-Parkinson-White patterns, abnormal QRS duration $(>120 \mathrm{~ms})$ or abnormal QTc prolongations ( $>450 \mathrm{~ms}$ ), and Q/ST/T segment abnormalities suggestive of acute or chronic ischemia.

\section{Statistical Analysis}

We calculated descriptive statistics for each predictor variable, stratified by the presence or absence of TTE findings. Chi-square and $t$-tests were used to test associations between categorical or continuous variables and TTE findings using a significance level of 0.05 and two-sided hypothesis testing. To identify a robust set of predictors of the primary outcome, we used multivariate logistic regression with the LASSO (Least Absolute Shrinkage and Selection Operator) to fit a parsimonious model. ${ }^{22}$ The LASSO selects variables and shrinks the associated coefficients to avoid overfitting. ${ }^{23-25}$ We then used a bootstrap to generate confidence intervals for coefficient estimates. Cases with missing echocardiography reports were excluded from the analysis. Bootstrap results were summarized as the percentage of bootstrap iterations in which each variable's coefficient was (1) chosen and negative, (2) shrunk to zero, or (3) chosen and positive.

We assessed different weighting schemes to generate a risk score from significant variables identified by regression modeling. These included weighting by regression coefficients rounded to the nearest integer and simple summation of the presence or absence of each variable.

Based on these results, a predictive score was developed to risk stratify patients on their probability of major, clinically significant findings on TTE. The sensitivity and specificity of a score of zero to predict findings on TTE was calculated. For confidence intervals, we used Wilson's method for binomial confidence intervals. ${ }^{26}$ The receiver operating characteristic (ROC) curve and its associated area under the curve (AUC) were calculated, and a confidence interval for the AUC was obtained through bootstrap resampling with 2,000 iterations. As part of our sensitivity analyses, we also calculated the ROC curve and AUC after excluding the patients with a known history of $\mathrm{CHF}$ and significant finding on TTE. Data analyses were performed in $\mathrm{R}^{27}{ }^{7}$ Two sensitivity analyses were performed: (1) we used multiple imputation to impute 1,000 complete data sets and then used the same LASSO methodology as with the complete data to assess whether incorporating missing data changed the results; and (2) we simulated a conventional troponin assay by raising the positive threshold for hs-TnT to $>30 \mathrm{pg} / \mathrm{mL}$ (corresponding to the limit of detection for conventional troponin). ${ }^{28}$

\section{RESULTS}

\section{Characteristics of Study Subjects}

Patient screening occurred from April 2013 to September 2016. There were 6,930 patients who met eligibility criteria, of whom 3,686 (53\%) consented and enrolled in the study (Figure 1). Of these, $995(27 \%)$ received TTE. The mean age of patients receiving TTE was 74 years; $55 \%$ were male. Characteristics of patients obtaining and not obtaining TTE are presented in Appendix Table 2. Patients who received TTE were more likely to be older, have abnormal heart sounds, abnormal EKGs, elevated hs-TnT, elevated NT-proBNP, and have a history of CHF. Of the 995 subjects receiving TTE, 215 (21.6\%) had a major, clinically significant finding.

\section{Main Results}

Univariate analysis identified 14 variables significantly associated with major findings on TTE. These included male gender, shortness of breath, abnormal heart sounds, history of renal failure, diabetes, CHF, CAD, abnormal ECG, and elevated cardiac biomarkers, among others (Table 1). The most common major finding on TTE was regional wall motion abnormality, followed by reduced left ventricular ejection fraction (Table 2). Of the 995 patients who received TTE, 20 (2\%) were discharged directly from the ED, 444 (45\%) were observed, and 531 (53\%) were admitted. On average, patients who received TTE had a longer length of stay than did those that did not (3.4 days vs 1.9 days).

LASSO multivariable logistic regression produced five predictors associated with major findings on TTE: (1) history of $C H F$, (2) history of CAD, (3) abnormal ECG, 4) hs-TnT above 14 $\mathrm{pg} / \mathrm{mL}$, and 5) NT-proBNP above $125 \mathrm{pg} / \mathrm{mL}$ (Table 3).

These five high-risk clinical variables retained their importance after multivariate analysis and form the ROMEO score. The sensitivity and specificity of a ROMEO score of zero for excluding major findings on TTE was $99.5 \%$ (95\% Cl: 97.4\%$99.9 \%)$ and $15.4 \%$ (95\% Cl: 13.0\%-18.1\%), respectively. Patients with a ROMEO score of 0 were at very low risk of having a major finding on TTE: 0.8\% (95\% Cl: 0.02\%-4.5\%; Appendix Table 3). Only one out of 121 patients with none of the ROMEO criteria was found to have a major finding on TTE (regional wall motion abnormality). Patients with a score of one or more were at moderate-to-high risk of having a major finding $(7.3 \%$ to $55.6 \%)$.

There was a linear relationship between the ROMEO score and probability of major findings on TTE (Appendix Figure 1). The AUC was 0.77 (95\% Cl=0.72-0.79) indicating good accuracy of the combination of the five high-risk clinical variables to predict major findings on TTE (Appendix Figure 2). After excluding the 72 patients with known CHF and significant findings on TTE, the AUC was similar: 0.73 (95\% Cl: 0.69-0.77). There were 139 patients with at least one missing variable (14\%; Appendix Table 4). A multiple imputation sensitivity analysis identified the same five high-risk clinical variables in $85 \%$ of imputations.

There were 253 patients with high-sensitivity troponin levels between 15 and $30 \mathrm{pg} / \mathrm{mL}$ (inclusive). Using a higher hs-TnT threshold (>30 pg/mL) to simulate a conventional troponin assay again identified the same five high-risk variables along with shortness of breath as a potential sixth variable though with an odds ratio approaching unity (Appendix Table 5). The ROMEO score would have missed two additional patients with major findings if the troponin cutoff were raised to $30 \mathrm{pg} / \mathrm{mL}$ from $14 \mathrm{pg} / \mathrm{mL}$, ie, it would have identified 212/215 (98.6\%) of the major findings rather than 214/215 (99.5\%). 
TABLE 1. Univariate Analysis: Clinical Variables Associated with Major Findings on Echocardiography after Syncope

\begin{tabular}{|c|c|c|c|c|c|}
\hline Clinical Variable & No. $(\%)$ & $\begin{array}{l}\text { Normal/minor findings on TTE } \\
\qquad(\mathrm{N}=780)\end{array}$ & Major Findings on TTE ( $\mathrm{N}=215)$ & Odds Ratio & $95 \% \mathrm{Cl}$ \\
\hline Age, mean (SD) & $74.1(9.1)$ & $73.9(8.9)$ & $74.8(9.9)$ & 1.01 & $(0.99,1.03)$ \\
\hline Male gender & $547(55)$ & $409(52)$ & $138(64)$ & 1.63 & $(1.19,2.22)$ \\
\hline \multicolumn{6}{|l|}{ Race } \\
\hline White & $813(82)$ & $639(82)$ & $174(81)$ & ref & ref \\
\hline Black & $146(15)$ & $110(14)$ & $36(17)$ & 1.20 & $(0.84,1.81)$ \\
\hline Other & $33(3)$ & $28(4)$ & $5(2)$ & 0.66 & $(0.25,1.72)$ \\
\hline Shortness of breath & $213(21)$ & $147(19)$ & $66(31)$ & 1.90 & $(1.35,2.68)$ \\
\hline Exertional syncope & $194(19)$ & $145(19)$ & $49(23)$ & 1.29 & $(0.89,1.86)$ \\
\hline Abnormal heart sounds & $133(13)$ & $87(11)$ & $46(21)$ & 2.15 & $(1.45,3.19)$ \\
\hline Chest discomfort & $86(9)$ & $63(8)$ & $23(11)$ & 1.41 & $(0.85,2.34)$ \\
\hline Near syncope & $296(30)$ & $216(28)$ & $80(37)$ & 1.55 & $(1.13,2.13)$ \\
\hline $\mathrm{SBP}>180 \mathrm{~mm} \mathrm{Hg}$ & $10(1)$ & $7(1)$ & $3(1)$ & 1.56 & $(0.40,6.08)$ \\
\hline $\mathrm{SBP}<90 \mathrm{~mm} \mathrm{Hg}$ & $42(4)$ & $35(4)$ & $7(3)$ & 0.72 & $(0.31,1.64)$ \\
\hline History of SCD in $1^{\text {st }}$ degree relative & $95(10)$ & $64(8)$ & $31(14)$ & 1.89 & $(1.19,2.99)$ \\
\hline History of hypertension & $683(69)$ & $520(67)$ & $163(76)$ & 1.56 & $(1.10,2.20)$ \\
\hline History of dysrhythmia & $250(25)$ & $173(22)$ & $77(36)$ & 1.95 & $(1.41,2.70)$ \\
\hline History of renal failure & $119(12)$ & $78(10)$ & $41(19)$ & 2.11 & $(1.40,3.20)$ \\
\hline History of diabetes & $266(27)$ & $191(24)$ & $75(35)$ & 1.65 & $(1.19,2.28)$ \\
\hline History of CHF & $153(15)$ & $81(10)$ & $72(33)$ & 4.33 & $(3.01,6.24)$ \\
\hline History of $C A D$ & $304(31)$ & $193(25)$ & $111(52)$ & 3.24 & $(2.37,4.42)$ \\
\hline Abnormal ECG & $611(61)$ & $437(56)$ & $174(81)$ & 4.08 & $(2.74,6.07)$ \\
\hline History of reduced EF & $35(4)$ & $13(2)$ & $22(10)$ & 6.71 & $(3.32,13.56)$ \\
\hline History of structural heart disease & $159(16)$ & $101(13)$ & $58(27)$ & 2.48 & $(1.72,3.57)$ \\
\hline Hs-TnT (>14 pg/ml) & $479(48)$ & $330(42)$ & $149(69)$ & 3.6 & $(2.53,5.14)$ \\
\hline NT-proBNP (>125 pg/ml) & $698(70)$ & $509(65)$ & $189(88)$ & 5.82 & $(3.36,10.06)$ \\
\hline
\end{tabular}

Abbreviations: CAD, Coronary Artery Disease; CHF, Congestive Heart Failure; Cl: Confidence Interval. ECG, Electrocardiogram; EF, Ejection Fraction; Hs-TnT, high-sensitivity cardiac troponin T; mm Hg, millimeters of mercury; NT-proBNP: N-terminal pro B-type natriuretic peptide; SBP, Systolic Blood Pressure; SCD, Sudden Cardiac Death; SD, Standard Deviation; TTE, Transthoracic Echocardiography.

Those with a race of "White" were used as the reference standard to which "Black" or "Other" were compared with.

\section{DISCUSSION}

Older adults with syncope often present to the ED and undergo a variety of diagnostic tests, including TTE, and a significant proportion are admitted to the hospital. ${ }^{2}$ There is currently no standardized, evidence-based approach to guide TTE ordering for these patients. Using a large, prospective dataset of syncope patients, we sought to develop a risk-stratification tool to help clinicians identify which syncope patients would be at very low risk for clinically significant findings on TTE. We found that in the absence of these five high-risk clinical variables, the rate of significant findings on TTE in our sample was less than 1\%. All five high-risk variables included in the tool remained predictive in our sensitivity analyses, speaking to the robustness of our model.

Other retrospective, and smaller prospective, studies have identified a combination of low-risk criteria including: a normal ECG alone, ${ }^{15}$ a normal physical exam and normal ECG, ${ }^{14,17}$ a negative cardiac history and normal ECG. ${ }^{16} \mathrm{Han}$ et al. performed a chart review of 241 patients presenting to the ED with syncope and identified three risk factors for abnormal TTE findings using multiple logistic regression: age, abnormal ECG, and BNP greater than $100 \mathrm{pg} / \mathrm{mL} .{ }^{13}$ While these studies' results are generally consistent with ours, the retrospective nature and small sample size of these studies limit the generalizability of these results. Thus, using a large, multicenter prospective dataset, we derived a clinical decision instrument (the ROMEO 
TABLE 2. List of Major, Clinically Significant

Echocardiogram Findings, $n=215$

\begin{tabular}{lc}
\hline Major Finding & Frequency, No. (\%) \\
\hline Regional wall motion abnormalities & $118(11.9)$ \\
\hline $\begin{array}{l}\text { Reduced Ejection Fraction (either <45\% or qualitative } \\
\text { "severe LV dysfunction") }\end{array}$ & $71(7.1)$ \\
\hline Right ventricular dysfunction/strain & $23(2.3)$ \\
\hline $\begin{array}{l}\text { Severe aortic stenosis (<1 cm²) } \\
\text { Severe pulmonary hypertension (eg, severely elevated }\end{array}$ & $20(2.0)$ \\
\hline PA systolic pressure) & $15(1.5)$ \\
\hline $\begin{array}{l}\text { Severe aortic regurgitation or severe mitral stenosis/regurgitation } \\
\text { (qualitative) }\end{array}$ & $14(1.4)$ \\
\hline \begin{tabular}{l} 
Hypertrophic cardiomyopathy with outflow tract obstruction \\
\hline Obstructive physiology (large pericardial effusion, atrial myxoma)
\end{tabular} & $4(0.4)$ \\
\hline $\begin{array}{l}\text { Abbreviations: cm, centimeter; LV, left ventricular; PA, pulmonary artery. } \\
\text { (Sum of individual findings greater than } 215 \text { due to some subjects having more than one } \\
\text { finding.) }\end{array}$
\end{tabular}

score) to determine which older adults with syncope are at very low risk for major, clinically significant findings on TTE.

Our results add to the recent American College of Cardiology/American Heart Association/Heart Rhythm Society guidelines on the management of syncope which recommend TTE in "selected patients presenting with syncope if structural heart disease is suspected. ${ }^{\prime 18}$ Our risk-stratification tool offers a simple, standardized approach to determine specifically when to defer TTE testing.

Our findings can guide clinicians in deciding when to obtain TTE for ED syncope patients in the following way: Older adults presenting with syncope or near-syncope to the ED who have none of the ROMEO criteria are at extremely low risk for clinically significant findings on TTE and thus need not undergo such testing solely because of the syncopal event. Patients who have only one or more high-risk clinical variables are at higher risk (7.3\%-56\%) of significant TTE findings. In this subset, other factors, (eg, physician gestalt, recent previous echocardiography, patient preference, availability of echocardiography) can help guide TTE ordering. Patients with a greater number of high-risk variables may benefit from a more urgent echocardiographic evaluation.

Although on average, patients undergoing TTE had a longer length of stay than those that did not, this finding does not necessarily imply that ordering a TTE was the cause of the increased length of stay. It is possible that this positive association was due to greater underlying medical complexity or acuity of illness that resulted in a greater likelihood of admission/ observation, and in turn, a greater length of stay.

Prior to implementation, our results should be externally validated in other clinical settings. In the interim, this risk-stratification tool may be used by clinicians, in conjunction with clinical judgement, to help guide the appropriate use of TTE in older adults presenting with syncope.
TABLE 3. Clinical Variables associated with Major Findings on Echocardiography using Multivariate LASSO regression

\begin{tabular}{lcc}
\hline Clinical Variable & Odds Ratio & $95 \% \mathrm{Cl}$ \\
\hline History of CHF & 1.60 & $(1.02,2.57)$ \\
\hline Abnormal ECG & 1.53 & $(1.18,2.48)$ \\
\hline NT-proBNP>125 pg/ml & 1.34 & $(1.00,2.61)$ \\
\hline HS-TnT>14 pg/ml & 1.29 & $(1.00,2.03)$ \\
\hline History of CAD & 1.24 & $(1.00,1.96)$ \\
\hline Age & 1.00 & $(1.00,1.00)$ \\
\hline Male gender & 1.00 & $(1.00,1.43)$ \\
\hline Abnormal heart sounds & 1.00 & $(1.00,1.55)$ \\
\hline Exertional syncope & 1.00 & $(1.00,1.26)$ \\
\hline Shortness of breath & 1.00 & $(1.00,1.67)$ \\
\hline Chest pain & 1.00 & $(1.00,1.18)$ \\
\hline Near syncope & 1.00 & $(1.00,1.43)$ \\
\hline Family history of SCD & 1.00 & $(1.00,2.06)$ \\
\hline SBP > 180 mm Hg & 1.00 & $(1.0082,1.00)$ \\
\hline SBP < 90 mm Hg & 1.00 & $(1.00,1.55)$ \\
\hline History of hypertension & 1.00 & $(1.00,1.00)$ \\
\hline History of dysrhythmia & $1.00,1.20)$ \\
\hline History of renal failure & & \\
\hline History of diabetes & & \\
\hline Abbreviations: CAD, Coronary Artery Disease; CHF, Congestive Heart Failure; Cl, Confidence \\
Interval; ECG, Electrocardiogram; Hs-TnT, high-sensitivity cardiac troponin T; mm Hg, millimeters \\
of mercury; NT-proBNP, N-terminal pro B-type natriuretic peptide; SBP, Systolic Blood Pressure; \\
\hline SCD, Sudden Cardiac Death.
\end{tabular}

Our study has certain limitations. As we only enrolled patients 60 years and older, our findings may not necessarily be valid in younger populations of syncope patients. However, structural heart disease is less common in younger patients and is generally more of a concern for clinicians when evaluating syncope patients in the older age range. ${ }^{29}$ In our study, $47 \%$ of eligible patients declined to participate and thus sampling bias may have occurred. TTEs were ordered at the discretion of treating providers, which was likely subject to physician, institutional, and regional variation; the prevalence of major TTE findings may be lower in the overall cohort than in patients who received TTE. Prior TTE reports were not available; therefore, we were not able to determine if these major findings were previously known. Importantly, we did not perform an internal or external validation of the ROMEO score due to time and resource constraints. Thus, this study represents a derivation of the score solely and would require external validation prior to clinical implementation. Also, to calculate the ROMEO score, both an hs-TnT and NT-proBNP level must be obtained. Thus, the cost savings of any potential 
reduction in TTE ordering may be partially offset by the costs of increased laboratory testing. Lastly, hs-TnT assays are not currently widely available in hospitals in the United States; earlier generation cardiac troponin assays may not be a perfect substitute for hs-TnT assays. Our sensitivity analysis using an elevated threshold for hs-TnT attempted to mitigate this limitation and resulted in similar findings.

In summary, this risk-stratification tool, using five simple criteria, could help clinicians determine which older adult syncope patients can safely forgo TTE. If validated, this tool could help optimize resource utilization, and increase the value of healthcare for patients presenting with syncope.

\section{Acknowledgments}

The authors would like to thank the research assistants at all 11 sites who enrolled patients and collected data for this study.

Disclosures: Dr. Adler has received research funding from Roche. Dr. Bastani has received research funding from Radiometer and Portola and has been a consultant for Portola. Dr. Baugh has received advisory board and speaker's fees from Roche, research funding from Janssen and Boehringer Ingelheim, and consulting and advisory board fees from Janssen. Dr. Casterino has received funding from Astra Zeneca. Dr. Clark has received research funding from Radiometer, Ortho Clinical Trials, Janssen, Pfizer, NIH, Portola, Biocryst, Glaxo Smith Klein, Hospital Quality Foundation, and Abbott. She is a consultant for Portola, Janssen, and Hospital Quality Foundation. Dr. Diercks is a consultant for Siemens, Janssen, and Roche has received institutional research support from Novartis, Ortho Scientific, and Roche. Dr. Hollander has received research funding from Alere, Siemens, Roche, Portola, and Trinity. Dr. Hollander has also received royalties from UpToDate. Dr. Nishijima has received an honorarium from Pfizer. Dr. Storrow is a consultant for Siemens and Quidel, has received speaking fees from MCM Education, and is on the Data and Safety Monitoring Board for Trevena. Dr. Sun is a consultant for Medtronic. The other authors report no relevant conflicts of interest.

Funding: This project was supported by the National Heart, Lung, and Blood Institute of the National Institutes of Health under Award Number R01 HL111033. Dr. Probst is supported by the National Heart, Lung, and Blood Institute of the National Institutes of Health under Award Number K23HL132052-02. The content is solely the responsibility of the authors and does not necessarily represent the official views of the National Institutes of Health. Roche Diagnostics supplied the high-sensitivity troponin-T assays. The sponsoring organizations had no role in the design and conduct of the study; collection, management, analysis, and interpretation of the data; and preparation, or review of the manuscript.

\section{References}

1. Sun BC, Emond JA, Camargo CA, Jr. Characteristics and admission patterns of patients presenting with syncope to U.S. emergency departments, 1992-2000. Acad Emerg Med. 2004;11(10):1029-1034. doi: 10.1197/j. aem.2004.05.032

2. Probst MA, Kanzaria HK, Gbedemah M, Richardson LD, Sun BC. National trends in resource utilization associated with ED visits for syncope. Am Emerg Med. 2015;33(8):998-1001. doi: 10.1016/j.ajem.2015.04.030.

3. Kapoor WN, Karpf M, Maher Y, Miller RA, Levey GS. Syncope of unknown origin. The need for a more cost-effective approach to its diagnosis evaluation. JAMA. 1982;247(19):2687-2691. doi: 10.1001/jama.247.19.2687.

4. Pires LA, Ganji JR, Jarandila R, Steele R. Diagnostic patterns and temporal trends in the evaluation of adult patients hospitalized with syncope. Arch Intern Med. 2001;161(15):1889-1895. doi: 10.1001/archinte.161.15.1889.

5. Quinn JV, Stiell IG, McDermott DA, Sellers KL, Kohn MA, Wells GA. Derivation of the San Francisco Syncope Rule to predict patients with shortterm serious outcomes. Ann Emerg Med. 2004;43(2):224-232. doi: 10.1016/ S0196064403008230.

6. Linzer M, Yang EH, Estes NA, 3rd, Wang P, Vorperian VR, Kapoor WN. Diag nosing syncope. Part 1: Value of history, physical examination, and electrocardiography. Clinical Efficacy Assessment Project of the American College of Physicians. Ann Intern Med. 1997;126(12):989-996. doi: 10.7326/0003-4819126-12-199706150-00012.

7. Linzer M, Yang EH, Estes NA, 3rd, Wang P, Vorperian VR, Kapoor WN Diagnosing syncope. Part 2: Unexplained syncope. Clinical Efficacy Assessment Project of the American College of Physicians. Ann Intern Med. 1997:127(1):76-86. doi: 10.7326/0003-4819-127-1-199707010-00014.

8. Sun BC, Emond JA, Camargo CA, Jr. Direct medical costs of syncope-related hospitalizations in the United States. Am J Cardiol. 2005;95(5):668-671. doi: 10.1016/j.amjcard.2004.11.013

9. American College of Cardiology Foundation. Appropriate Use Criteria Task F, American Society of Echocardiography, American Heart Association, et al. ACCF/ASE/AHA/ASNC/HFSA/HRS/SCAI/SCCM/SCCT/SCMR 2011 Appropriate Use Criteria for Echocardiography. A Report of the American College of Cardiology Foundation Appropriate Use Criteria Task Force, American Society of Echocardiography, American Heart Association, American Society of Nuclear Cardiology, Heart Failure Society of America, Heart Rhythm Society, Society for Cardiovascular Angiography and Interventions, Society of Critical Care Medicine, Society of Cardiovascular Computed Tomography, and Society for Cardiovascular Magnetic Resonance Endorsed by the American College of Chest Physicians. J Am Coll Cardiol. 2011;57(9):1126-1166. doi: 10.1016/j.echo.2010.12.008.

10. Maganti K, Rigolin VH, Sarano ME, Bonow RO. Valvular heart disease: diagnosis and management. Mayo Clin Proc. 2010;85(5):483-500. doi: 10.4065/ mcp.2009.0706

11. Mendu ML, McAvay G, Lampert R, Stoehr J, Tinetti ME. Yield of diagnostic tests in evaluating syncopal episodes in older patients. Arch Intern Med. 2009;169(14):1299-1305. doi: 10.1001/archinternmed.2009.204.

12. Madeira CL, Craig MJ, Donohoe A, Stephens JR. Things we do for no reason: echocardiogram in unselected patients with syncope. J Hosp Med. 2017;12(12):984-988. doi: http://dx.doi.org/10.12788/jhm.2864.

13. Han SK, Yeom SR, Lee SH, et al. Transthoracic echocardiogram in syncope patients with normal initial evaluation. Am J Emerg Med. 2017;35(2):281-284. doi: 10.1016/j.ajem.2016.10.078

14. Chang NL, Shah P, Bajaj S, Virk H, Bikkina M, Shamoon F. Diagnostic yield of echocardiography in syncope patients with normal ECG. Cardiol Res Pract. 2016;2016:1251637. doi: http://dx.doi.org/10.1155/2016/1251637.

15. Anderson KL, Limkakeng A, Damuth E, Chandra A. Cardiac evaluation for structural abnormalities may not be required in patients presenting with syncope and a normal ECG result in an observation unit setting. Ann Emerg Med. 2012;60(4):478-84.e1. doi: 10.1016/j.annemergmed.2012.04.023.

16. Sarasin FP, Junod AF, Carballo D, Slama S, Unger PF, Louis-Simonet M. Role of echocardiography in the evaluation of syncope: a prospective study. Heart. 2002;88(4):363-367. doi: 10.1136/heart.88.4.363

17. Recchia D, Barzilai B. Echocardiography in the evaluation of patients with syncope. J Gen Intern Med. 1995;10(12):649-655. doi: 10.1007/BF02602755.

18. Shen WK, Sheldon RS, Benditt DG, et al. ACC/AHA/HRS guideline for the evaluation and management of patients With syncope: executive summary: A report of the American College of Cardiology/American Heart Association Task Force on Clinical Practice Guidelines and the Heart Rhythm Society. J Am Coll Cardiol. 2017:2017(70(5)):620-663

19. Chiu DT, Shapiro NI, Sun BC, Mottley JL, Grossman SA. Are echocardiography, telemetry, ambulatory electrocardiography monitoring, and cardiac enzymes in emergency department patients presenting with syncope useful tests? A preliminary investigation. J Emerg Med. 2014;47(1):113-118. doi: 10.1016/j.jemermed.2014.01.018.

20. Sun BC, Costantino $G$, Barbic $F$, et al. Priorities for emergency department syncope research. Ann Emerg Med. 2014;64(6):649-55.e2. doi: 10.1016/j. annemergmed.2014.04.014.

21. Sun BC, Derose SF, Liang LJ, et al. Predictors of 30-day serious events in older patients with syncope. Ann Emerg Med. 2009;54(6):769-778.e1-5. doi: 10.1016/j.annemergmed.2009.07.027.

22. Tibshirani R. Regression shrinkage and selection via the lasso. J R Stat Soc. 1996;58(1):267-288.

23. Friedman J, Hastie T, Tibshirani R. He Elements of Statistical Learning;Vol 1. New York, NY: Springer-Verlag; 2001

24. Friedman J, Hastie T, Tibshirani R. Regularization paths for generalized linear models via coordinate descent. J Stat Softw. 2010;33(1):1-22. doi: 10.18637/ jss.v033.i01.

25. James G, Witten D, Hastie T, Tibshirani R. An Introduction to Statistical Learning; Vol 112. New York, NY: Springer-Verlag; 2013

26. WilsonEB. Probable inference, the law of succession, and statistical inference. $J$ Am Stat Assoc. 1927;22(158):209-212. doi: 10.1080/01621459.1927.10502953.

27. R Core Team (2018). R: A language and environment for statistical computing. R Foundation for Statistical Computing, Vienna, Austria. URL http://www.R-project.org/.

28. Chew DP, Zeitz C, Worthley M, et al. Randomized comparison of high-sensitivity troponin reporting in undifferentiated chest pain assessment. Circ Cardiovasc Qual Outcomes. 2016;9(5):542-553. doi: 10.1161/CIRCOUTCOMES.115.002488

29. Chen RS, Bivens MJ, Grossman SA. Diagnosis and management of valvular heart disease in emergency medicine. Emerg Med Clin North Am. 2011;29(4):801-10, vii. doi: 10.1016/j.emc.2011.08.001. 